\title{
Impact of non-cardiovascular disease burden on thirty-day hospital readmission in heart failure patients
}

\author{
Valentina Kutyifa, John Rice, Roy Jones, Andrew Mathias, Ayhan Yoruk, \\ Katherine Vermilye, Brent Johnson, Robert Strawderman, Charles Lowenstein \\ University of Rochester Medical Center, Rochester, New York, United States
}

\begin{abstract}
Background: Little is known about the impact of non-cardiovascular disease (CVD) burden on 30-day readmission in heart failure (HF) patients. The aim of the study was to assess the role of non-CVD burden on 30-day readmission in HF patients.

Methods: We analyzed the effect of non-CVD burden by frequency of ICD-9 code categories on readmissions of patients discharged with a primary diagnosis of HF. We first modeled the probability of readmission within 30 days as a function of demographic and clinical covariates in a randomly selected training dataset of the total cohort. Variable selection was carried out using a bootstrap LASSO procedure with 1000 bootstrap samples, the final model was tested on a validation dataset. Adjusted odds ratios and confidence intervals were reported in the validation dataset.

Results: There were a total of 6228 HF hospitalizations, 1523 (24\%) with readmission within 30 days of discharge. The strongest predictor for 30-day readmissions was any hospital admission in the prior year $(p<0.001)$. Cardiovascular risk factors did not enter the final model. However, digestive system diseases increased the risk for readmission by $17 \%$ for each diagnosis $(p=0.046)$, while respiratory diseases and genitourinary diseases showed a trend toward a higher risk of readmission $(p=0.07$ and $p=0.09$, respectively). Non-CVDs out-competed cardiovascular covariates previously reported to predict readmission.

Conclusions: In patients with HF hospitalization, prior admissions predicted 30-day readmission. Diseases of the digestive system also increase 30-day readmission rates. Assessment of non-CVD burden in HF patients could serve as an important risk marker for 30-day readmissions. (Cardiol J 2018; 25, 6: 691-700)
\end{abstract}

Key words: heart failure readmission, non-cardiovascular disease burden, predictive model

\section{Introduction}

Heart failure (HF) is a significant health issue affecting approximately 5.7 million adults in the United States with estimated annual costs of over $\$ 30$ billion [1]. About one quarter of the patients discharged with a primary diagnosis of $\mathrm{HF}$ are readmitted within 30 days [2]. Hospital readmis- sions are associated with significant additional cost and adverse clinical outcomes. The Center for Medicare and Medicaid Services (CMS) Hospital Readmissions Reduction Program provides financial incentives to hospitals to reduce readmissions for these patients. As a result, significant attention and effort has been put in to reducing readmission of $\mathrm{HF}$ patients.

Address for correspondence: Valentina Kutyifa, MD, PhD, Heart Research Follow-up Program, Cardiology Division, University of Rochester Medical Center, 265 Crittenden Blvd., PO Box 653, Rochester, NY 14642, USA, tel: 585-275-2676, fax: 585-273-5283, e-mail: Valentina.Kutyifa@heart.rochester.edu or valentina.heart@alumni.harvard.edu 
Identifying HF patients at high risk for hospital readmission is crucial for targeting interventions to reduce readmissions. Several prior studies predicted 30-day readmissions, although the predictive accuracy of these models is limited [3, 4]. Since the outcome of interest is 30-day readmission for any cause, it is possible that non-cardiovascular risk factors play an important role. While prior studies looked at socioeconomic and psychosocial characteristics [5], there is limited information on non-cardiovascular risk factors predicting 30-day readmission.

Therefore, in the present study, we aimed to develop a predictive model for HF readmissions including parameters reflecting non-cardiovascular disease (CVD) burden, using a novel statistical bootstrap LASSO approach for variable selection. In addition, we examined the specific reasons, including non-cardiovascular diagnoses, for which HF patients are readmitted within 30 days following $\mathrm{HF}$ hospitalization discharge.

\section{Methods}

This is a retrospective analysis of hospital admission data for patients discharged from the University of Rochester Medical Center (Rochester, NY) with a primary diagnosis of HF (ICD-9 codes starting with 428 , plus $402.01,402.11$, 402.91, 404.01, 404.03, 404.11, 404.13, 404.91, 404.93). Patients under the age of 18 and patients that passed away before discharge were excluded from this study.

\section{Definitions and end points}

An index admission for each patient was defined as an admission with a primary discharge diagnosis of HF between January $1^{\text {st }}, 2007$, and June $30^{\text {th }}, 2014$, as previously described [6]. Recurrent readmissions beyond the 30 -day readmission window were included in the analysis. The primary outcome was 30-day hospital readmission for any cause, defined as a hospitalization for any cause within 30 days after discharge from the index $\mathrm{HF}$ admission. Records on hospital admissions between January $1^{\text {st }}, 2006$ and December 31, 2006 were used to derive the number of hospital admissions in the prior year for patients discharged in 2007.

When assessing the ability of non-cardiovascular diagnoses at the time of $\mathrm{HF}$ admission to predict subsequent readmissions, we used the following ICD-9 code categories: 1 ) infectious and parasitic diseases, 2) neoplasms, 3) endocrine, nutritional and metabolic diseases, and immunity disorders, 4) diseases of the blood and blood-forming organs, 5) mental disorders, 6) diseases of the nervous system, 7) diseases of the respiratory system, 8) diseases of the digestive system, 9) diseases of the genitourinary system, 10) diseases of the skin and subcutaneous tissue, and 11) diseases of the musculoskeletal system and connective tissue. We assessed the total number of each diagnosis for a patient within these categories at the time of the index HF discharge as a covariate to predict 30-day readmission. Specific reasons for readmissions within 30 days following the HF index admission were also analyzed using the same ICD-9 categories ( $<1 \%$ was labeled "Other").

\section{Statistical analysis}

Statistical analyses were performed using the $\mathrm{R}$ software [7]. The summary statistics in Tables 1 and 2 were calculated using the entire data set of $6228 \mathrm{HF}$ hospitalizations. The significance tests and p-values for these tables were calculated using the generalized estimating equations (GEE) methodology [8], which takes into account the correlation between multiple admissions for a single patient. For each covariate, a logistic regression GEE model was fit to the outcome containing that covariate only: the p-value displayed in the tables is for the robust test of no effect for that covariate on probability of 30-day readmission.

For the purposes of multivariate analyses, the data was first randomly split on the basis of patient ID into training and validation sets: the training set was used for purposes of model selection, while the validation set was used for calculation of coefficient estimates and standard errors for the selected model. We used multivariable GEE regression models to estimate the probability of 30-day readmission. Our final model, with three standard CMS covariates (age, gender, and race) is shown in Table 3. For comparison, the same model without the three CMS covariates is shown in Table 4.

Variable selection was carried out using a bootstrap LASSO procedure: 1000 bootstrap samples were generated from the training set, sampling with replacement on the basis of patients' ID (repeated measurements on a subject were either all included or all excluded from each sample) [9]. For each of these samples, the LASSO procedure [10] was applied using the R package glmnet. The order in which the covariates entered the model was then recorded, with the covariate entering first considered to be the most predictive. Figure 1A 
Table 1. Characteristics of patients and admissions by 30-day hospital readmission.

\begin{tabular}{lcccc}
\hline Parameters & $\begin{array}{c}\text { All } \\
\text { admissions }\end{array}$ & $\begin{array}{c}\text { Admissions } \\
\text { with 30-day } \\
\text { readmission }\end{array}$ & $\begin{array}{c}\text { Admissions } \\
\text { without 30-day } \\
\text { readmission }\end{array}$ & P \\
\hline Number of patients & 1999 & 366 & 1633 \\
Age [years]** & $68.34(15.5)$ & $68.08(16.1)$ & $68.41(15.4)$ & 0.606 \\
Female gender** & $1668(0.41)$ & $346(0.44)$ & $1322(0.40)$ & 0.103 \\
White race** & $3063(0.75)$ & $582(0.73)$ & $2481(0.76)$ & 0.139 \\
Number of admissions in prior year* & $1.48(2.36)$ & $2.51(3.48)$ & $1.14(1.73)$ & $<0.001$ \\
Diagnoses on admission* & $16.52(6.4)$ & $18.14(6.88)$ & $16.00(6.14)$ & $<0.001$ \\
Admission type emergency & $4326(0.69)$ & $1144(0.75)$ & $3182(0.68)$ & $<0.001$ \\
Length of stay [days]* & $8.99(17.98)$ & $11.01(21.03)$ & $8.34(16.82)$ & $<0.001$ \\
Disposition to home & $5192(0.83)$ & $1205(0.79)$ & $3987(0.85)$ & $<0.001$ \\
Cardiovascular disease burden of admission & & & $1629(0.35)$ & $<0.001$ \\
Hypertension & $2069(0.33)$ & $440(0.29)$ & $1954(0.42)$ & 0.407 \\
Hyperlipidemia & $2567(0.41)$ & $613(0.40)$ & 1954 \\
Cardiomyopathy & $1498(0.24)$ & $343(0.23)$ & $1155(0.25)$ & 0.206 \\
Left bundle branch block & $369(0.06)$ & $62(0.04)$ & $307(0.07)$ & $<0.001$ \\
Arrhythmias & $302(0.05)$ & $55(0.04)$ & $247(0.05)$ & 0.009 \\
Atrial fibrillation & $2511(0.40)$ & $619(0.41)$ & $1892(0.4)$ & 0.798 \\
Smoking & $2179(0.35)$ & $533(0.35)$ & $1646(0.35)$ & 0.993 \\
End stage renal disease & $478(0.08)$ & $155(0.10)$ & $323(0.07)$ & $<0.001$ \\
\hline
\end{tabular}

Results are shown as count (proportion), except those with a * are mean (standard deviation). **Data are for all patients included, not for admissions.

Table 2. Non-cardiovascular disease burden of admissions by 30-day hospital readmission.

\begin{tabular}{lcccc}
\hline Parameters & $\begin{array}{c}\text { All } \\
\text { admissions }\end{array}$ & $\begin{array}{c}\text { Admissions } \\
\text { with 30-day } \\
\text { readmission }\end{array}$ & $\begin{array}{c}\text { Admissions } \\
\text { without 30-day } \\
\text { readmission }\end{array}$ & $\begin{array}{c}\text { P } \\
\text { Number of patients }\end{array}$ \\
Infectious and parasitic diseases & 1999 & 366 & 1633 \\
Neoplasms & $959(0.15)$ & $306(0.20)$ & $653(0.14)$ & $<0.001$ \\
Endocrine, nutritional and metabolic diseases, & $529(0.05)$ & $97(0.06)$ & $232(0.05)$ & 0.036 \\
and immunity disorders & $5413(0.87)$ & $1354(0.89)$ & $4059(0.86)$ & 0.012 \\
Diseases of the blood and blood-forming organs & $2272(0.36)$ & $654(0.43)$ & $1618(0.34)$ & $<0.001$ \\
Mental disorders & $2766(0.44)$ & $748(0.49)$ & $2018(0.43)$ & $<0.001$ \\
Diseases of the nervous system & $2051(0.33)$ & $524(0.34)$ & $1527(0.32)$ & 0.196 \\
Diseases of the respiratory system & $2988(0.48)$ & $841(0.55)$ & $2147(0.46)$ & $<0.001$ \\
Diseases of the digestive system & $2441(0.39)$ & $671(0.44)$ & $1770(0.38)$ & $<0.001$ \\
Diseases of the genitourinary system & $4031(0.65)$ & $1134(0.74)$ & $2897(0.62)$ & $<0.001$ \\
Diseases of the skin and subcutaneous tissue & $610(0.10)$ & $175(0.11)$ & $435(0.09)$ & 0.014 \\
Diseases of the musculoskeletal system and & $1352(0.22)$ & $342(0.22)$ & $1010(0.21)$ & 0.444 \\
connective tissue & & & &
\end{tabular}

Results are shown as counts (proportions) of admissions for which any diagnoses in that category were present on admission.

shows boxplots depicting the distribution of variable importance determined by this method, where variables are ordered from top to bottom by the mean order of entry. Also computed for each bootstrap sample was the out-of-bag (OOB: all subjects in the training set not included in that particular 
Table 3. Predictors of 30-day hospital readmission in a multivariate logistic regression model in the validation dataset.

\begin{tabular}{|c|c|c|c|c|}
\hline Covariate & Estimate & $95 \%$ LCL & $95 \%$ UCL & $\mathbf{P}$ \\
\hline Number prior admissions (past year) & 1.21 & 1.14 & 1.27 & $<0.001$ \\
\hline Log length of stay & 1.14 & 1.00 & 1.30 & 0.057 \\
\hline Emergency admission & 0.93 & 0.70 & 1.25 & 0.640 \\
\hline Discharge to home & 0.78 & 0.57 & 1.08 & 0.132 \\
\hline Number of respiratory disease diagnoses & 1.14 & 0.99 & 1.31 & 0.070 \\
\hline Number of digestive disease diagnoses & 1.17 & 1.00 & 1.36 & 0.046 \\
\hline Number of genitourinary disease diagnoses & 1.11 & 0.98 & 1.26 & 0.092 \\
\hline Age & 0.99 & 0.99 & 1.00 & 0.173 \\
\hline Female gender & 1.00 & 0.78 & 1.28 & 0.991 \\
\hline White race & 0.91 & 0.69 & 1.20 & 0.504 \\
\hline
\end{tabular}

Area under the ROC curve (AUC): 0.643; LCL — lower confidence limit; UCL — upper confidence limit

Table 4. Predictors of 30-day hospital readmission in a multivariate logistic regression model in the validation dataset without Center for Medicare and Medicaid Services (CMS) covariates (age, gender, and race).

\begin{tabular}{lcccc}
\hline Covariate & Estimate & 95\% LCL & 95\% UCL & P \\
\hline Log length of stay & 1.15 & 1.01 & 1.315 & $\mathbf{0 . 0 3 3}$ \\
Number prior admissions (past year) & 1.22 & 1.16 & 1.285 & $<\mathbf{0 . 0 0 1}$ \\
Emergency admission & 0.92 & 0.70 & 1.208 & 0.551 \\
Discharge to home & 0.84 & 0.61 & 1.142 & 0.263 \\
Number of respiratory disease diagnoses & 1.13 & 0.98 & 1.304 & 0.085 \\
Number of digestive disease diagnoses & 1.16 & 0.99 & 1.349 & 0.054 \\
Number of genitourinary disease diagnoses & 1.10 & 0.97 & 1.234 & 0.137 \\
\hline
\end{tabular}

Area under the ROC curve (AUC): 0.642; $L C L$ - lower confidence limit; UCL — upper confidence limit

bootstrap sample) area under the ROC curve (AUC) [11] as a function of degrees of freedom in the model (Fig. 1B). The degrees of freedom reflect the number of covariates included and is a measure of model complexity. By using the OOB subjects to calculate AUC, we are able to get a more unbiased estimate than would be obtained from using the data twice (first to fit the model and then to compute AUC). The point at which the OOB AUC was maximized was used to determine an approximate number of covariates that should be included in the model. Suppose this criterion indicates that using $d$ covariates is best; then, to construct our final model, we select the $d$ covariates that had the lowest average rank of entry into the LASSO model (i.e., at the top of Fig. 1A), where the average is calculated for each covariate over all bootstrap samples.
To obtain a predicted probability of readmission for each patient, we used the model selected by the bootstrap LASSO method on the training set, along with the standard CMS covariates, with coefficients estimated by applying that model to the training set. These estimated coefficients were then used to produce predictions for both the training and validation sets. The $95 \%$ confidence intervals were obtained by running an interceptonly GEE model for each quantile separately under working independence correlation structure and applying the expit transform to the endpoints of a 95\% confidence interval for the estimated logit of the mean (Fig. 2). We also assessed reason for readmission in the study by looking at the record for the next admission for hospitalizations that led to a 30-day readmission (Table 5). The table shows the count and percentage of admissions for each 


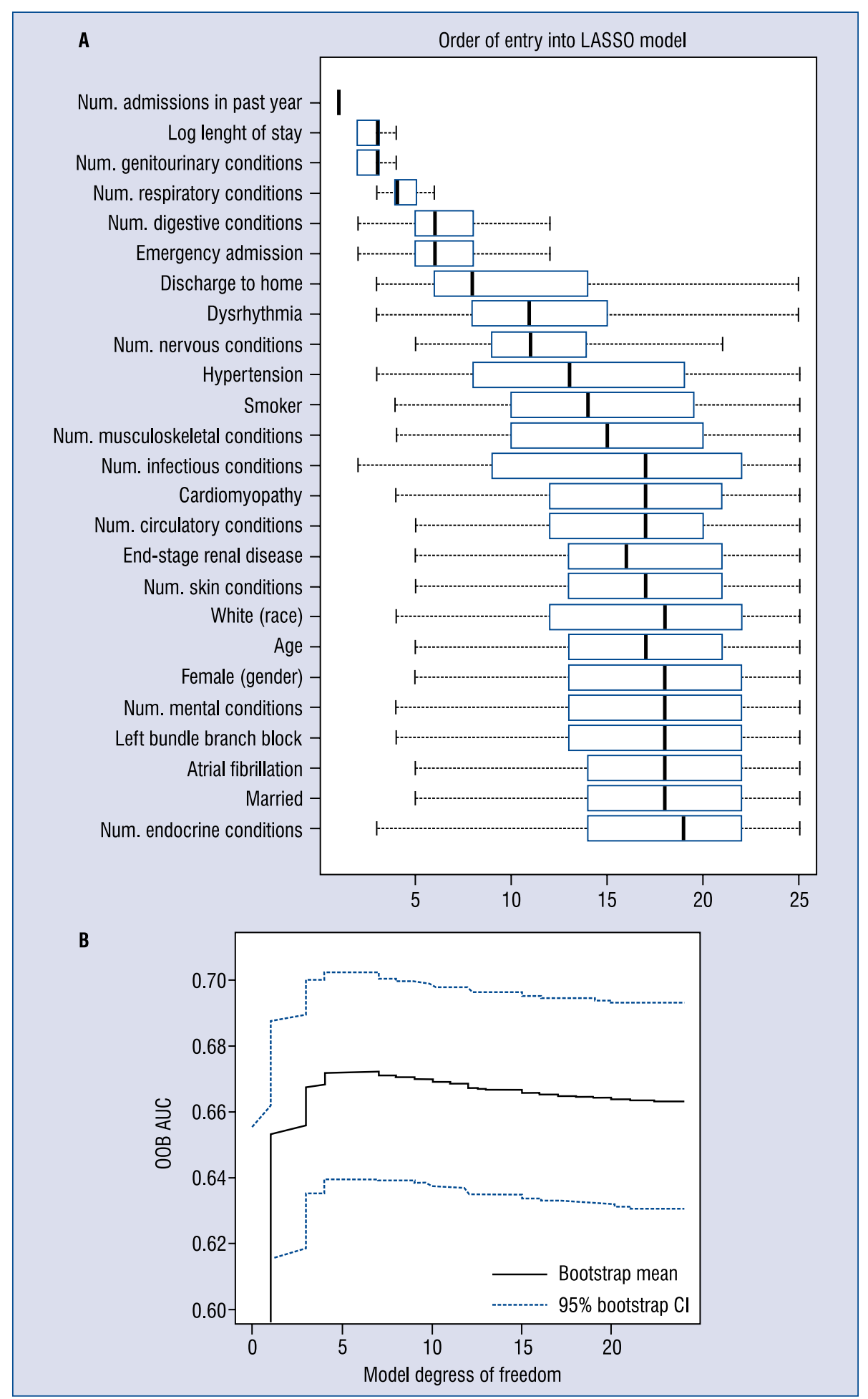

Figure 1. A. Order of entry into the LASSO model in the training dataset. Panel A shows the distribution of order of entry into the LASSO model across 1000 bootstrap samples. Each boxplot corresponds to a covariate, which have been sorted for this figure by mean rank, so that the covariate appearing at the top would have, on average, entered the model earliest, etc; B. selection of covariates in the final model in the validation dataset. Panel B. shows the model's predictive accuracy as a function of the degrees of freedom, or the number of variables included in the model. This is obtained for each bootstrap sample by predicting the outcomes for those hospitalizations that were not included in the fitting procedure for that sample (the out-of-bag, or OOB, sample), then computing the area under the ROC curve (AUC) for each value of the LASSO tuning parameter. This produces a curve of the type shown in this figure for each bootstrap sample. This figure was obtained by averaging the values of these curves at each point. The $95 \%$ confidence limits are obtained from the 0.025 and 0.975 quantiles of these curves at each point. The point at which the AUC is maximized gives an idea of a reasonable choice for the number of covariates to include in the final model; $\mathrm{Cl}$ - confidence interval 


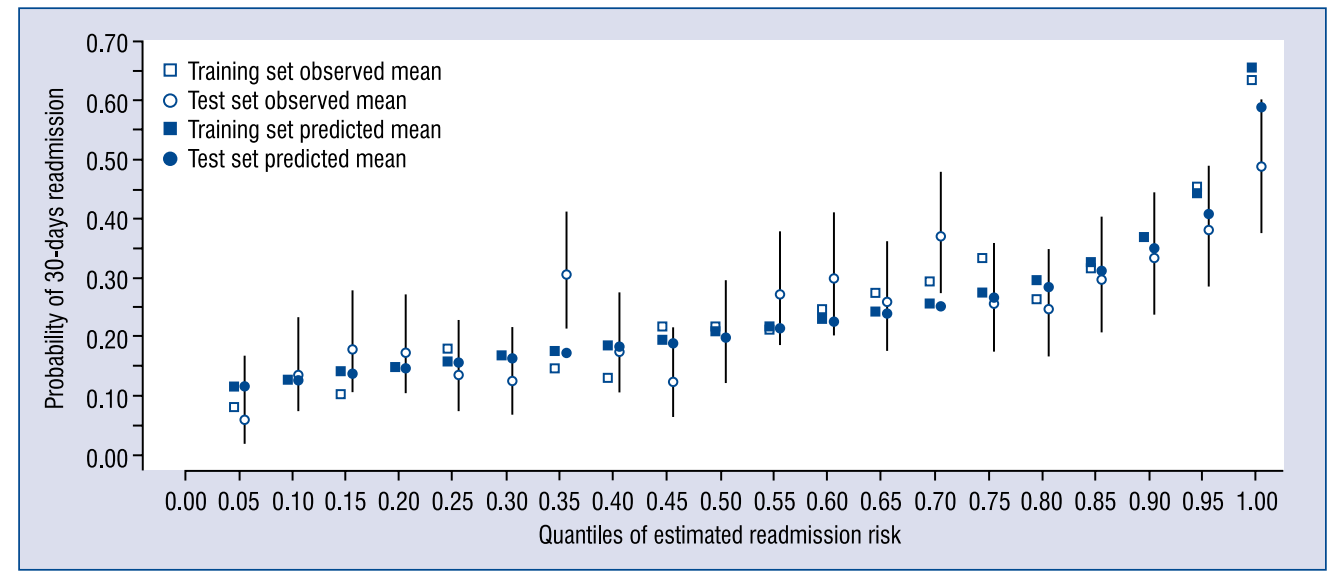

Figure 2. Calibration plot of the estimation of 30-day readmission rates in the training and validation datasets. This figure shows a calibration plot for the final selected model (shown in Table 4). This is obtained by first splitting the data along quantiles of the estimated risk of readmission separately for the training and validation data sets. For each such subgroup, the empirical mean of the outcome (open symbols) and the mean of the predicted probabilities (filled symbols) were calculated. Also shown are $95 \%$ binomial confidence intervals for the empirical outcome means.

Table 5. Specific reasons for readmission within 30 days in the total cohort by ICD-9 codes.

\begin{tabular}{lcc}
\hline Diagnosis & $\begin{array}{c}\text { Number } \\
\text { of readmissions }\end{array}$ & $\begin{array}{c}\text { Percentage } \\
(\%)\end{array}$ \\
\hline Diseases of the circulatory system & 883 & $58.0 \%$ \\
Diseases of the respiratory system & 106 & $7.0 \%$ \\
Diseases of the genitourinary system & 83 & $5.4 \%$ \\
Injury and poisoning & 82 & $5.4 \%$ \\
Diseases of the digestive system & 79 & $5.2 \%$ \\
Symptoms, signs, and ill-defined conditions & 59 & $3.9 \%$ \\
Endocrine, nutritional and metabolic diseases, and immunity disorders & 54 & $3.5 \%$ \\
External causes of injury and supplemental classification & 54 & $3.5 \%$ \\
Infectious and parasitic diseases & 50 & $3.3 \%$ \\
Diseases of the mental system & 16 & $1.1 \%$ \\
Other & 57 & $3.7 \%$ \\
\hline
\end{tabular}

ICD-9 code category of the primary diagnosis on readmission.

\section{Results}

There were 6652 hospital discharges with a primary diagnosis of HF between January $1^{\text {st }}$, 2007 , and June $30^{\text {th }}, 2014$, at the University of Rochester Medical Center. A total of 32 patients who were younger than 18 years were excluded from the analysis. In the remaining cohort of 6620 hospitalizations, 392 patients died in the hospital, leaving us with 6228 total HF discharges from a total of 4061 unique patients (Fig. 3). There were a total of 1523 (24\%) of these HF discharges that led to a subsequent readmission within 30 days.

\section{Baseline clinical characteristics}

Baseline characteristics of the 4061 patients' age, gender, and race and other characteristics of the 6228 total HF discharges are displayed in Tables 1 and 2 . The mean age of the study population was 68 years. Women made up $41 \%$ of the sample; $75 \%$ were white. The mean length of stay was 9 days. Patients with 30-day readmission were more often admitted to the emergency room $(p<0.001)$, 


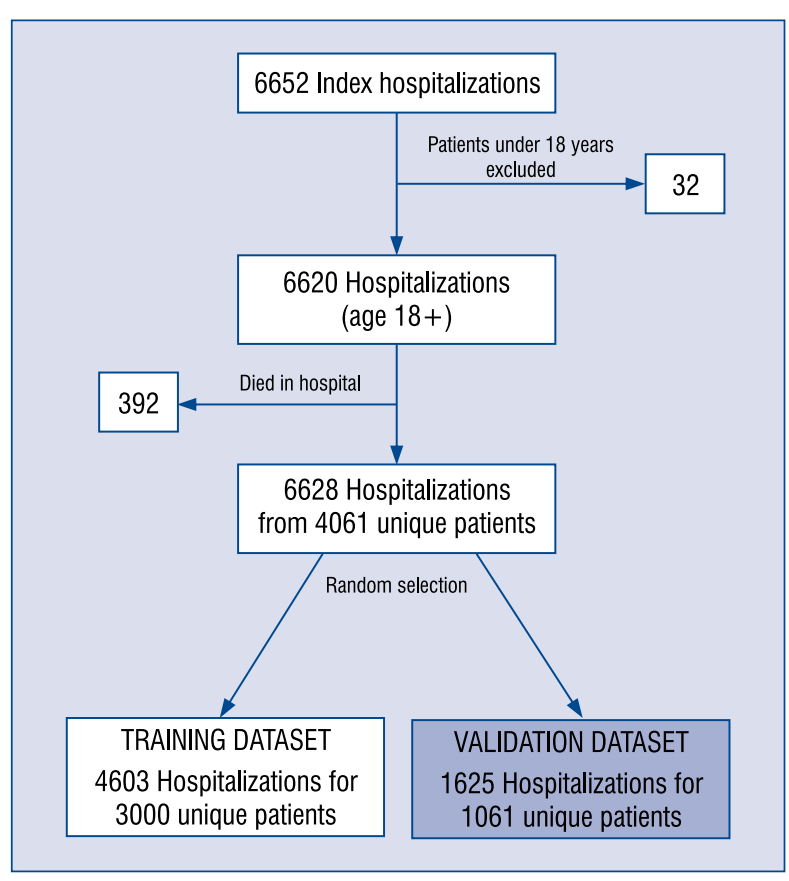

Figure 3. Study flowchart.

they had a longer length of stay (11 vs. 8 days, $\mathrm{p}<0.001)$, and they were less likely to be discharged to home ( $<<0.001)$. Hypertension ( $<<0.001)$, left bundle branch block $(\mathrm{p}<0.001)$, and arrhythmias $(\mathrm{p}<0.01)$ were less prevalent in patients with readmissions, while end stage renal disease was more common in readmitted patients $(\mathrm{p}=0.001)$.

We observed a high prevalence of non-CVD burden in our HF cohort. Table 2 displays the non-CVD burden of the $6228 \mathrm{HF}$ discharges in our study. Over $80 \%$ of the admissions had additional diagnoses of endocrine, nutritional, metabolic or immunity disorders, and $65 \%$ of the admissions mentioned diseases of the genitourinary system. Other common diagnoses included respiratory disease (48\%), mental disorders (44\%), digestive disease (39\%), diseases of the blood (36\%), and diseases of the nervous system (33\%). HF admissions linked to subsequent 30 -day readmission were more often accompanied with concomitant infectious diseases, diseases of the blood, mental disorders, and respiratory, digestive, and genitourinary ailments $(\mathrm{p}<0.001$ for all).

\section{Predictors of 30-day readmissions in the training set}

Using the LASSO procedure in our training set, the number of hospital admissions in the prior year (in the 365 days prior to the index admission) emerged as the strongest predictor of 30-day re- admissions, followed by log length of stay and the number of concomitant genitourinary conditions at admission. Other relevant predictors were the number of respiratory conditions, the number of digestive disorders, emergency admission, and discharge to home (Fig. 1A). These covariates were similarly predictive of the end point in univariate analysis (Tables 1,2). Using OOB AUC (averaged across all bootstrap samples) as a criterion, we determined that our model should include these seven covariates (i.e., appearing at the top of Fig. 1A), along with the three standard CMS covariates. Interestingly, no cardiovascular diagnoses and none of the CMS variables were among the seven most predictive covariates.

\section{Predictors of 30-day readmissions in the validation set}

In our validation set (Table 3 ), one additional hospital admission in the prior year was associated with a $21 \%$ increase in the risk of 30 -day readmission $(\mathrm{p}<0.001$; Fig. $4 \mathrm{~A})$, and each digestive system diagnosis increased the risk for readmission by $17 \%$ ( $\mathrm{p}<0.05)$. Log-transformed length of stay $(p<0.06)$ showed a trend toward a higher risk of readmission, with longer stays being associated with greater risk of readmission. Respiratory and digestive diseases were similarly associated with higher risk or readmission with increasing burden of these co-morbidities ( $p=0.07$ and $p=0.09$, respectively; Fig. 4B). Emergency admission and discharge to home performed less strongly in predicting 30-day readmission in the validation set. Removing the CMS covariates from our model led to only a very small reduction in AUC (AUC 0.643 with CMS covariates, AUC 0.642 without CMS covariates; Table 4). The model including only CMS covariates had a substantially lower AUC of 0.542 .

A calibration plot estimating 30 -day readmission rates in the training and validation set is displayed in Figure 2. This figure shows, for both the training and the validation sets, the average predicted probability of readmission within 30 days and the empirical proportion of 30-day readmissions, stratified by quantiles of the predicted risk of readmission. We see good general agreement between both observed and predicted risks and between the training and test sets, with three exceptions. At the 0.35 and 0.70 quantiles, the test set shows some underestimation of the risk of readmission (although the training set does not); at the highest end of the risk spectrum, risk seems to be overestimated by the model, both in the training and the test sets. There is also a gradual trend in the 


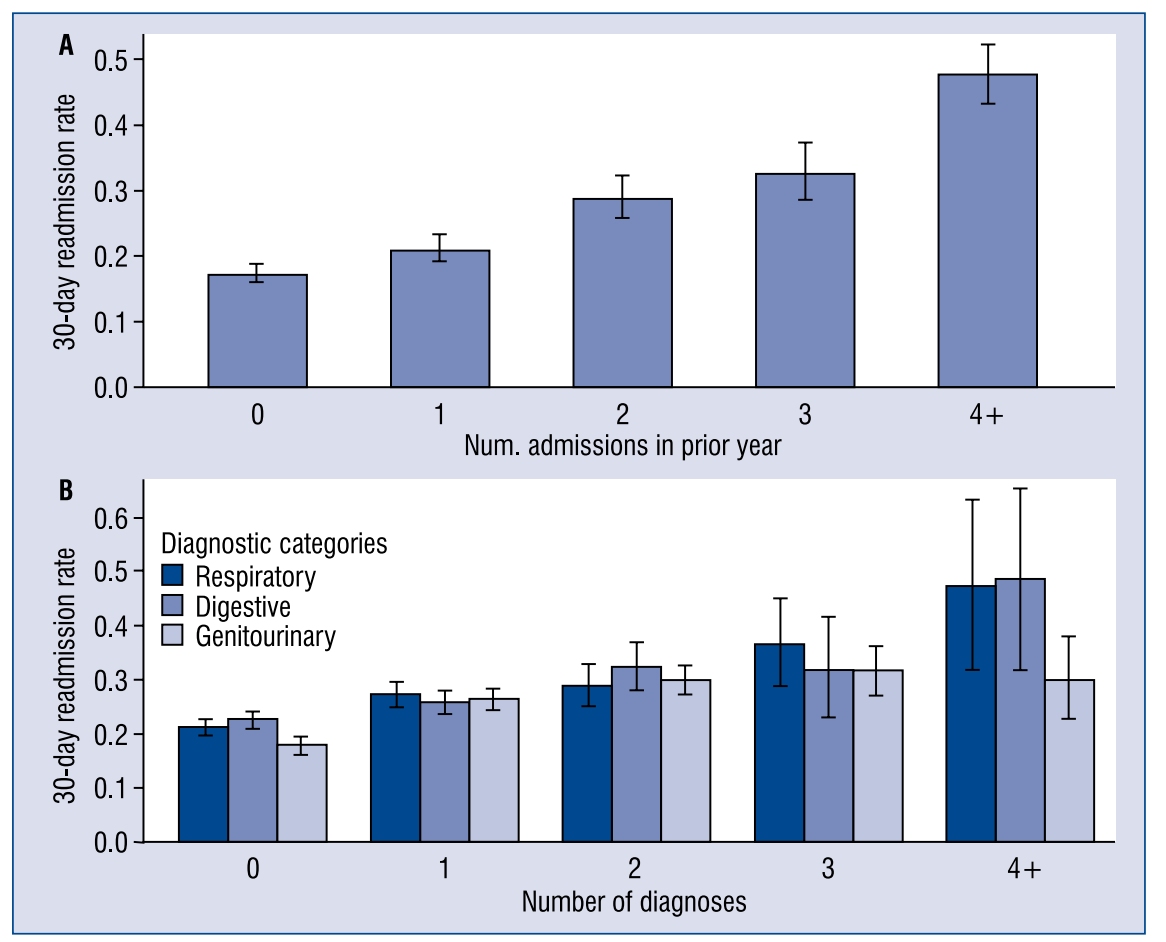

Figure 4. The rate of 30-day readmission by A. Prior admissions; B. Number of respiratory diagnoses, number of digestive diagnoses, and number of genitourinary diagnoses. These figures display bar plots of the 30-day readmission rate within subgroups of the study population defined by some covariates. This rate is calculated as the sample mean of the binary outcome variable within each subgroup.

means of the predicted probabilities where those of the training set are greater than those in the test set, with the difference increasing for higher quantiles of risk. A similar calibration plot using only the CMS covariates shows good agreement however the overall risk profile is much flatter in comparison to that seen in Figure 2, and also demonstrates comparatively poor agreement with the observed risk of readmission (data not shown).

\section{Cause-specific 30-day readmissions}

Assessing specific reasons for 30-day readmission, we found that over half of the readmissions $(58 \%)$ were due to diseases of the circulatory system, primarily due to HF. However, a significant proportion of patients were admitted due to non-circulatory conditions, including respiratory disease $(7 \%)$, such as pneumonia, bronchitis, or acute respiratory failure, genitourinary diseases (5\%), such as kidney failure, injuries (5\%), and diseases of the digestive system (5\%) (Table 5).

\section{Discussion}

In the present study, we used a novel statistical method to derive and rank importance of predic- tors of 30-day hospital readmission in patients hospitalized for HF in a training set that was tested in an independent validation set. Prior hospital admission emerged as a significant predictor of 30-day hospital readmission. Importantly, noncardiovascular comorbidities played a role predicting 30-day readmissions, including diseases of the digestive system, and diseases of the respiratory and genitourinary system. These data suggest the importance of diverse risk assessment of patients with $\mathrm{HF}$ admission including the assessment of non-cardiovascular risk factors. Confirming our observations, we also revealed that 30-day readmissions often occurred due to non-cardiovascular causes. Assessment and proper management of non-cardiovascular co-morbidities could potentially reduce 30 -day hospital readmissions.

Our findings are novel and add to the estimation of readmission risk by taking into account non-CVD burden. Interestingly, in our model, which included length of stay and prior hospital admissions, non-cardiovascular comorbidities out-competed other covariates such as that race, gender, and age, that had been previously reported to be important in predicting readmission [12]. Amajor reason why non-cardiovascular comorbidities 
predict hospital readmission is that a large percentage of patients who were originally hospitalized for $\mathrm{HF}$ are readmitted to the hospital for other reasons. This has previously been suggested by Krumholz et al. [13] using the National Medicare, and Davis et al. [14] in the Healthcare Cost and Utilization Project State Inpatient Databases. Our results are confirmatory, $42 \%$ of the readmissions were due to non-cardiovascular causes. Our study has important clinical relevance, identifying and properly managing non-CVD burden could potentially reduce readmissions and related healthcare costs and avoiding adverse outcomes [15-17].

Several studies suggested that atrial fibrillation and hypertension could be associated with the risk of hospital readmission $[18,19]$. However, we found that patients with hypertension were less likely to be readmitted than those without. This could be explained by the fact that our center is a tertiary referral hospital and treats a disproportionately high percentage of advanced HF patients who are unlikely to have hypertension due to severe systolic dysfunction. It could be that patients with hypertension more often have with diastolic HF and those patients typically have worse outcomes due to the limited available treatment options [20,21].

In our study, diseases of the digestive system, as well as respiratory and genitourinary diseases, were identified as predictors of 30-day hospital readmissions. Structural and functional changes to the gastrointestinal system are closely related to the process of HF, with intricate pathophysiology often blurring the line between primary and secondary events. Also, it is has been shown that HF-related congestion of the gastrointestinal system can lead to further HF exacerbation events through potential medication malabsorption [22, 23]. The impact of genitourinary disease including renal dysfunction on the progression of $\mathrm{HF}$ has been well described in the literature [24, 25]. Patients with renal dysfunction are at risk for accelerated atherosclerosis, hypertension, and fluid retention, all of which have unfavorable consequences for HF patients. Furthermore, these patients are less likely to be on guideline directed optimal medical therapy for systolic dysfunction due to concern for exacerbation of renal function or the development of hyperkalemia. Finally, cardiorenal syndrome has been described as a separate entity in HF patients, associated with adverse clinical outcomes [26]. However, our study has not been designed to specifically prove causality between these non-cardiovascular disorders and readmissions, and further studies are warranted.
Despite the inclusion of non-cardiovascular factors, our predictive model is still insufficient to explain a significant proportion of readmissions. This is similar to prior predictive models [27-29], despite including information on mental health, cognitive function, or socioeconomic factors [30-33]. We have to acknowledge that 30-day readmissions are complex, multifaceted events that might include post-hospital syndrome [34, 35], or frailty [36].

Our study has limitations. First, this is a single-center study, and therefore, the results may not be generalizable. Our study is retrospective and non-randomized, and might carry referral bias, selection bias and confounding. Lack of follow-up for patients who died within 30 days or who were readmitted to another hospital system may have affected our analysis.

\section{Conclusions}

In a single-center analysis of 30-day readmissions following hospitalization for HF, digestive disorders along with prior admissions were predictive of 30-day readmission, while diseases of the respiratory and genitourinary system, and length of stay showed a trend for association. More than $40 \%$ of readmissions in our cohort were due to noncardiovascular diagnoses. Our study highlights the need for assessment of non-CVD burden when estimating 30-day readmission risk in $\mathrm{HF}$ patients, as well as identifying preventive measures targeting non-CVD burden to reduce 30 -day readmissions.

\section{References}

1. Go A, Mozaffarian D, Roger V, et al. Heart disease and stroke statistics - 2014 update. Circulation. 2014; 129(3): e28-e292, doi: 10.1161/01.cir.0000441139.02102.80.

2. Ross JS, Chen J, Lin Z, et al. Recent national trends in readmission rates after heart failure hospitalization. Circ Heart Fail. 2010; 3(1): 97-103, doi: 10.1161/CIRCHEARTFAILURE.109.885210, indexed in Pubmed: 19903931.

3. Dharmarajan K, Wang Y, Lin Z, et al. Association of changing hospital readmission rates with mortality rates after hospital discharge. JAMA. 2017; 318(3): 270-278, doi: 10.1001/ /jama.2017.8444, indexed in Pubmed: 28719692.

4. Krumholz H, Lin Z, Keenan P, et al. Relationship between hospital readmission and mortality rates for patients hospitalized with acute myocardial infarction, heart failure, or pneumonia. JAMA. 2013; 309(6): 587-593, doi: 10.1001/jama.2013.333.

5. Rathore SS, Masoudi FA, Wang Y, et al. Socioeconomic status, treatment, and outcomes among elderly patients hospitalized with heart failure: findings from the National Heart Failure Project. Am Heart J. 2006; 152(2): 371-378, doi: 10.1016/j. ahj.2005.12.002, indexed in Pubmed: 16875925. 
6. Kutyifa V, Moss AJ, Klein H, et al. Use of the wearable cardioverter defibrillator in high-risk cardiac patients: data from the Prospective Registry of Patients Using the Wearable Cardioverter Defibrillator (WEARIT-II Registry). Circulation. 2015; 132(17): 1613-1619, doi: 10.1161/CIRCULATIONAHA.115.015677, indexed in Pubmed: 26316618.

7. Team RCR. A Language and Environment for Statistical Computing. Vienna, Austria: R Foundation for Statistical Computing. 2015.

8. Fitzmaurice GM, Laird NM, Ware H. Applied longitudinal analysis Hoboken, N J. 2004.

9. Efron B. Bootstrap Methods: Another Look at the Jackknife. Ann Statist. 1979; 7(1): 1-26, doi: 10.1214/aos/1176344552.

10. Tibshirani R. Regression shrinkage and selection via the Lasso. J Roy Stat Soc B Met. 1996; 58: 267-88.

11. Bristow M, Saxon L, Boehmer J, et al. Cardiac-Resynchronization therapy with or without an implantable defibrillator in advanced chronic heart failure. N Engl J Med. 2004; 350(21): 2140-2150, doi: 10.1056/nejmoa032423.

12. Keenan PS, Normand SLT, Lin Z, et al. An administrative claims measure suitable for profiling hospital performance on the basis of 30-day all-cause readmission rates among patients with heart failure. Circ Cardiovasc Qual Outcomes. 2008; 1(1): 29-37, doi: 10.1161/CIRCOUTCOMES.108.802686, indexed in Pubmed: 20031785.

13. Krumholz HM, Chen YT, Wang Y, et al. Predictors of readmission among elderly survivors of admission with heart failure. Am Heart J. 2000; 139(1 Pt 1): 72-77, indexed in Pubmed: 10618565.

14. Davis JD, Olsen MA, Bommarito K, et al. All-Payer analysis of heart failure hospitalization 30-day readmission: comorbidities matter. Am J Med. 2017; 130(1): 93.e9-93.e28, doi: 10.1016/j. amjmed.2016.07.030, indexed in Pubmed: 27592085.

15. Chen LM, Jha AK, Guterman S, et al. Hospital cost of care, quality of care, and readmission rates: penny wise and pound foolish? Arch Intern Med. 2010; 170(4): 340-346, doi: 10.1001/ archinternmed.2009.511, indexed in Pubmed: 20177036.

16. Tokatli A, Ural D. Previous hospitalizations predict both hospital readmissions and mortality in patients with heart failure. Cardiol J. 2016; 23(2): 224-224, doi: 10.5603/cj.2016.0020.

17. Swindle JP, Chan WW, Waltman Johnson K, et al. Evaluation of mortality and readmissions following hospitalization with heart failure. Curr Med Res Opin. 2016 [Epub ahead of print]: 1-11, doi: 10.1080/03007995.2016.1205972, indexed in Pubmed: 27348501.

18. Desai A, Stevenson L. Rehospitalization for heart failure. Circulation. 2012; 126(4): 501-506, doi: 10.1161/circulationaha.112.125435.

19. Sherer AP, Crane PB, Abel WM, et al. Predicting heart failure readmissions. J Cardiovasc Nurs. 2016; 31(2): 114-120, doi: 10.1097/JCN.0000000000000225, indexed in Pubmed: 25513988.

20. Rigolli M, Whalley GA. Heart failure with preserved ejection fraction. J Geriatr Cardiol. 2013; 10(4): 369-376, doi: 10.3969/j. issn.1671-5411.2013.04.011, indexed in Pubmed: 24454331.

21. Sharma K, Kass DA. Heart failure with preserved ejection fraction: mechanisms, clinical features, and therapies. Circ Res. 2014; 115(1): 79-96, doi: 10.1161/CIRCRESAHA.115.302922, indexed in Pubmed: 24951759.

22. Vasko MR, Cartwright DB, Knochel JP, et al. Furosemide absorption altered in decompensated congestive heart failure. Ann Intern Med. 1985; 102(3): 314-318, indexed in Pubmed: 3970471.
23. Sica DA, Sica DA. Drug absorption in congestive heart failure: impact on management. Prog Cardiovasc Nurs. 1999; 14(1): 30-32, indexed in Pubmed: 10431317.

24. Smilde TDJ, Hillege HL, Voors AA, et al. Prognostic importance of renal function in patients with early heart failure and mild left ventricular dysfunction. Am J Cardiol. 2004; 94(2): 240-243, doi: 10.1016/j.amjcard.2004.03.075, indexed in Pubmed: 15246913.

25. Lang CC, Mancini DM. Non-cardiac comorbidities in chronic heart failure. Heart. 2007; 93(6): 665-671, doi: 10.1136/ /hrt.2005.068296, indexed in Pubmed: 16488925.

26. Liang KV, Williams AW, Greene EL, et al. Acute decompensated heart failure and the cardiorenal syndrome. Crit Care Med. 2008; 36(1 Suppl): S75-S88, doi: 10.1097/01. CCM.0000296270.41256.5C, indexed in Pubmed: 18158481.

27. Ross JS, Mulvey GK, Stauffer B, et al. Statistical models and patient predictors of readmission for heart failure: a systematic review. Arch Intern Med. 2008; 168(13): 1371-1386, doi: 10.1001/ /archinte.168.13.1371, indexed in Pubmed: 18625917.

28. Kansagara D, Englander H, Salanitro A, et al. Risk prediction models for hospital readmission: a systematic review. JAMA. 2011; 306(15): 1688-1698, doi: 10.1001/jama.2011.1515, indexed in Pubmed: 22009101.

29. Frizzell JD, Liang Li, Schulte PJ, et al. Prediction of 30-day all-cause readmissions in patients hospitalized for heart failure: comparison of machine learning and other statistical approaches. JAMA Cardiol. 2017; 2(2): 204-209, doi: 10.1001/jamacardio.2016.3956, indexed in Pubmed: 27784047.

30. Krumholz H, Bernheim S. The Role of Socioeconomic Status in Hospital Outcomes Measures. Ann Intern Med. 2015; 162(9): 670, doi: 10.7326/115-5087-2.

31. Priori SG, Blomstrom-Lundqvist C, Mazzanti A, et al. 2015 ESC Guidelines for the management of patients with ventricular arrhythmias and the prevention of sudden cardiac death: The Task Force for the Management of Patients with Ventricular Arrhythmias and the Prevention of Sudden Cardiac Death of the European Society of Cardiology (ESC). Endorsed by: Association for European Paediatric and Congenital Cardiology (AEPC). Eur Heart J. 2015; 36: 2793-2867.

32. Huynh Q, Negishi K, Blizzard L, et al. Predictive score for 30-day readmission or death in heart failure. JAMA Cardiol. 2016; 1(3): 362-364, doi: 10.1001/jamacardio.2016.0220.

33. Calvillo-King L, Arnold D, Eubank KJ, et al. Impact of social factors on risk of readmission or mortality in pneumonia and heart failure: systematic review. J Gen Intern Med. 2013; 28(2): 269-282, doi: 10.1007/s11606-012-2235-x, indexed in Pubmed: 23054925.

34. Mesquita ET, Cruz LN, Mariano BM, et al. Post-Hospital syndrome: a new challenge in cardiovascular practice. Arq Bras Cardiol. 2015; 105(5): 540-544, doi: 10.5935/abc.20150141, indexed in Pubmed: 26577722.

35. Krumholz HM. Post-hospital syndrome--an acquired, transient condition of generalized risk. N Engl J Med. 2013; 368(2): 100-102, doi: 10.1056/NEJMp1212324, indexed in Pubmed: 23301730.

36. Khan H, Kalogeropoulos AP, Georgiopoulou VV, et al. Frailty and risk for heart failure in older adults: the health, aging, and body composition study. Am Heart J. 2013; 166(5): 887-894, doi: 10.1016/j.ahj.2013.07.032, indexed in Pubmed: 24176445 . 